\title{
Identification of key transcription factors associated with cerebral ischemia-reperfusion injury based on gene-set enrichment analysis
}

\author{
YING-YING ZHANG ${ }^{1}$, KAI WANG ${ }^{1}$, YUN-E LIU ${ }^{1}$, WEI WANG ${ }^{2}$, AO-FEI LIU ${ }^{1}$, JI ZHOU ${ }^{1}$, \\ CHEN LI ${ }^{1}$, YI-QUN ZHANG ${ }^{1}$, AI-PING ZHANG ${ }^{1}$, JIN LV $^{1,3}$ and WEI-JIAN JIANG ${ }^{1}$ \\ ${ }^{1}$ Department of Vascular Neurosurgery, New Era Stroke Care and Research Institute, \\ The PLA Rocket Force Characteristic Medical Center, Beijing 100088; ${ }^{2}$ Shanghai Institute of Advanced \\ Immunochemical Studies, ShanghaiTech University, Shanghai 201210; ${ }^{3}$ Department of Nuclear and Radiation Injury, \\ The PLA Rocket Force Characteristic Medical Center, Beijing 100088, P.R. China
}

Received December 5, 2018; Accepted March 29, 2019

DOI: $10.3892 /$ ijmm.2019.4159

\begin{abstract}
Cerebral ischemia-reperfusion injury (CIRI) usually causes detrimental complications following reperfusion therapy in stroke patients. The present study systematically investigated the regulatory mechanism involved in the pathogenesis of CIRI using gene set enrichment analysis of the transient middle cerebral artery occlusion mouse stroke model. The results revealed a total of 13 CIRI-related transcription factors (TFs), including CCAAT enhancer binding protein b (Cebpb), Cebpa, early growth response-1, Fos, Rela, Jund, signal transduction and activator of transcription $5 \mathrm{a} / \mathrm{b}$, transformation related protein 53, GLI family zinc finger 2 (Gli2), Sp3, TF AP-2 a (Tfap2a) and spleen focus forming virus proviral integration oncogene (Spil). To the best of our knowledge, five TFs (Cebpa, Gli2, Sp3, Tfap2a and Spil) were the first to be reported associated with CIRI in the present study. The five novel CIRI-related TFs were mainly associated with pathways of inflammation and responses to reperfusion, including the tumor necrosis factor signaling pathway (Gli2, Spil and Tfap 2a, $\mathrm{P}=0.0035,0.0035$ and 0.048 , respectively), interleuking-17 signaling pathway (Cebpa, Gli2, Sp3, Spil and Tfap $2 a, \mathrm{P}=0.019,0.047,0.019,0.035$ and 0.005 , respectively) and fluid shear stress and atherosclerosis (Gli2, Sp3, Spil and Tfap $2 a, \mathrm{P}=0.047,0.046,0.013$ and 0.003 , respectively). These results may improve understanding of the potential molecular mechanism underlying the pathogenesis of CIRI at the genome-wide level.
\end{abstract}

Correspondence to: Dr Wei-Jian Jiang or Dr Jin Lv, Department of Vascular Neurosurgery, New Era Stroke Care and Research Institute, The PLA Rocket Force Characteristic Medical Center, 16 Xin-Wai Avenue, Xi-Cheng, Beijing 100088, P.R. China

E-mail: cjr.jiangweijian@vip.163.com

E-mail: 1vjin6630@hotmail.com

Key words: ischemic stroke, cerebral ischemia-reperfusion injury, transcription factor, gene-set enrichment analysis, functional pathway enrichment

\section{Introduction}

Cerebral ischemia-reperfusion injury (CIRI) is a complex pathophysiological process that occurs early in the restoration of blood supply following ischemia, and peaks at $\sim 24 \mathrm{~h}$ after reperfusion $(1,2)$. There are several factors that serve important roles in the pathogenesis of CIRI, including free radical formation, inflammatory cells infiltration, mitochondrial dysfunction and blood-brain-barrier disruption (3). These pathological events crucially rely on alterations in the transcription of various genes induced by ischemia and reperfusion. Numerous dysregulated genes associated with apoptosis, inflammation and metabolism were identified in a variety of ischemic stroke experimental models by high-throughput microarray or RNA-sequencing approaches $(4,5)$; however, the underlying regulatory mechanism of gene dysregulation associated with CIRI requires further investigation.

Studies have reported that the activation of transcription factors (TFs) induced by different cellular signal transduction pathways after ischemia serves a pivotal role in controlling gene expression and is closely associated with ischemic injury and neuronal survival $(6,7)$. Therefore, modulating the activity of CIRI-associated TFs, for example, via the recently reported RP105-phosphatidylinositol 3-kinase (PI3K)/protein kinase B (Akt) pathway (8), may be an effective strategy to disrupt CIRI. Studies have revealed that several TFs associated with neuronal injury are transcriptionally upregulated after focal ischemia, including interferon regulatory factor-1, nuclear factor- $\kappa \mathrm{B}(\mathrm{NF}-\kappa \mathrm{B})$, early growth response-1 (Egr1), CCAAT enhancer binding protein- $\beta$ (CEBP $\beta$ ), signal transduction and activator of transcription 3 (STAT3), and activating transcription factor-2 (9-13). However, additional TFs activities are thought to be regulated mainly by protein post-translational modifications, such as phosphorylation, which may not be investigated in traditional microarray expression analysis or experimental studies.

Considering that TFs regulate transcription by binding to specific regulatory elements upstream of the target genes, large-scale profiling of consensus TF binding sites (TFBS) from microarray expression data during ischemic brain injury can be another feasible option to comprehensively investigate 
the roles of TFs in cerebral ischemia. Several computational strategies were developed to predict TFs associated with cerebral ischemia, by detecting over-represented TFBS in the promoter regions of differentially expressed genes from microarray expression data (14-16). Although, to the best of our knowledge, few studies have focused on the TFs and their associated functions involved in CIRI after reperfusion via various pathways.

In the present study, we performed gene set enrichment analysis (GSEA) to determine the key TFs and their regulatory mechanisms of the biological pathways associated with CIRI. GSEA utilizes the whole-distribution testing model to identify the pre-defined TF-targeted gene-sets that are statistically enriched in CIRI group samples (17). In addition, the candidate TFs were re-calculated with Gene Ontology (GO) and Kyoto Encyclopedia of Genes and Genomes (KEGG) categories associated with CIRI, using the 'Post-Analysis' feature of the EnrichmentMap software (18). Our findings could provide important insight into the various transcriptional mechanisms associated with CIRI during ischemic stroke.

\section{Materials and methods}

Microarray data. Microarray assays were performed based on a previous transcriptional study of mouse ischemic tolerance in transient middle cerebral artery occlusion (tMCAO) stroke model, which included 224 samples with 6 experimental conditions $(19,20)$. In the present study, we investigated CIRI in mice suffering 45-min tMCAO or sham surgical procedure at 3 and $24 \mathrm{~h}$ after reperfusion ( $\mathrm{n}=4 /$ treatment/time), respectively (Table I). The original CEL files of the 45-min tMCAO group ( $\mathrm{n}=8, \mathrm{I} 3$ and $\mathrm{I} 24$ groups) and relevant sham surgery group ( $\mathrm{n}=8, \mathrm{~S} 3$ and $\mathrm{S} 24$ groups) were downloaded from the National Center of Biotechnology Information Gene Expression Omnibus (https://www.ncbi.nlm.nih.gov/geo/) via the accession number GSE32529, which was based on the platform of the GPL1261 [Mouse430_2] Affymetrix Mouse Genome 430 2.0 Array (Thermo Fisher Scientific, Inc.). Experimental analysis confirmed infarcts in tMCAO mice at 3 or $24 \mathrm{~h}$ after reperfusion, but not in sham-operated mice (19). It should be noted that we performed a bioinformatics study based on the public microarray data, and no mice were used for experimental analysis in the present study.

Data quality control and preprocessing. Data quality control and preprocessing were performed by the software packages of Bioconductor (v3.7, https://www.bioconductor.org/) based on the $\mathrm{R}$ statistical programming language (v3.5.0). Data quality was evaluated by RNA degradation plots using AffyRNAdeg algorithm in the affy package (v1.60.0) (21). The dataset showing a high slope of probe intensities from the $5^{\prime}$ to $3^{\prime}$ end, which serves as the quantitative indicator of the RNA degradation, was removed from our analysis prior to data preprocessing (Fig. S1A). For data preprocessing, we used the GC Robust Multi-array Average (gcRMA) algorithm in gcrma package (v2.54.0) to normalize raw expression data and generate the normalized gene expression intensity of the two data-sets with logarithmic transformation. Following normalization, the distributions of the normalized probe set intensities were evaluated by density histograms and boxplot (Fig. S1B and C). In the final step, the probe ID in normalized expression data was annotated to gene symbol based on the mouse4302cdf package (v2.18.0), and the data were exported to a formed text file ready for GSEA.

GSEA and leading-edge analysis. The gene set enrichment analysis and leading-edge analysis were performed using GSEA desktop application version 3.0 (Broad Institute), which employs predefined gene sets from the Gene Set Knowledgebase database (http://ge-lab.org/\#/data). In the present study, GSEA was performed using the collected gene-sets of TF target genes (mm_TF), GO (mm_GO) and metabolic pathways (mm_metabolic), with the following parameters: 1,000 gene set permutations, weighted enrichment statistics, gene set size between 15-500, and signal-to-noise metrics for ranking genes. Regulated gene-sets were considered statistically significant if false discovery rate (FDR) $\leq 0.1$ and nominal $\mathrm{P} \leq 0.01$. The GSEA-derived normalized enrichment score was used to determine the magnitude of up- or down-regulation of enriched gene-sets. Leading-edge analysis was performed after each GSEA to determine the core genes that have the highest impact on the biological process of a given gene-set.

For each database of gene sets, GSEA was respectively performed across two expression datasets: i) S3I3 dataset, which contains the expression data from the sham and tMCAO groups $3 \mathrm{~h}$ after reperfusion; ii) S24I24 dataset, which contains the expression data from the sham and tMCAO groups $24 \mathrm{~h}$ after reperfusion; and iii) I3I24 dataset, which contains the expression data from the $\mathrm{TMCAO}$ groups of 3 and $24 \mathrm{~h}$ after reperfusion.

Functional enrichment visualization and annotation. The GSEA results were visualized using EnrichmentMap (v3.1) plugin based on Cytoscape Desktop program (v3.6.1, https://cytoscape. $\mathrm{org} /$ ) (18). The enrichment results were mapped as a network of gene-sets (nodes) where the nodes represent statistically significant terms and the links (edges) represent the degree of gene-set similarity; combined Jaccard (50\%) and Overlap (50\%) metrics with the default cutoff of 0.375 were employed. For gene-sets annotation, the enriched gene-sets were grouped by AutoAnnotate software (v1.2, http://apps.cytoscape.org/apps/autoannotate) according to the Markov cluster (MCL) algorithm based on the edge weights of similarity coefficients and were automatically annotated using the WordCloud algorithm with the maximum of four words per label (22).

KEGG (https://www.genome.jp/kegg/) pathway enrichment analysis was performed using clusterProfiler package (v3.8.1) (23) and visualized via the ggplot2 package (v3.0.0) in $\mathrm{R}$ (24). The significant KEGG categories were identified with BH adjusted $\mathrm{P}<0.01$ and FDR $\mathrm{q}<0.05$.

Signature gene-sets post-analysis. Using the 'Post-Analysis' feature of the EnrichmentMap software, we constructed the transcriptional regulatory network associated with CIRI. The CIRI-related signature gene-sets were primarily defined as the gene-sets specifically enriched in the I 24 group by Venn analysis (http://bioinformatics.psb.ugent.be/webtools/Venn/) of the GSEA results between S3I3 and S24I24 datasets. The gmt file of I24-specific TFs signature gene-sets (mm_I24_TF) was created from the mm_TF gene-set database using custom 
Table I. Description of the tMCAO experimental groups.

\begin{tabular}{lllc}
\hline Group & Sample no. & Treatment & Time point (h) \\
\hline S3 & GSM805706 & Sham & 3 \\
S3 & GSM805707 & Sham & 3 \\
S3 & GSM805708 & Sham & 3 \\
S3 & GSM805709 & Sham & 3 \\
I3 & GSM805730 & tMCAO & 3 \\
I3 & GSM805731 & tMCAO & 3 \\
I3 & GSM805732 & tMCAO & 3 \\
I3 & GSM805733 & tMCAO & 3 \\
S24 & GSM805714 & Sham & 24 \\
S24 & GSM805715 & Sham & 24 \\
S24 & GSM805716 & Sham & 24 \\
S24 & GSM805717 & Sham & 24 \\
I24 & GSM805741 & tMCAO & 24 \\
I24 & GSM805742 & tMCAO & 24 \\
I24 & GSM805743 & tMCAO & 24 \\
I24 & GSM805744 & tMCAO & 24 \\
\hline
\end{tabular}

tMCAO, transient middle cerebral artery occlusion.

written $\mathrm{R}$ code. The transcriptional regulatory network was constructed based on the GSEA results of the GO or metabolic pathway network in EnrichmentMap, followed by post-analysis with CIRI-associated TFs signature gene-sets (mm_I24_TF), respectively. The edge weights, which represent the overlap between the signature gene-sets and enriched gene-sets in the network, were computed by Mann-Whitney U test with $\mathrm{P}=0.05$, and were visualized as pink edges with thickness indicating the degree of significance.

Microarray gene-expression analysis. The gene expression analysis of CIRI-associated TFs and their targeted genes were performed using linear regression models implanted in limma package (v3.38.2); P-values were calculated using an empirical Bayesian method, adjusted by Bonferroni correction (25). The pheatmap package (v1.0.10) was used to show the expression pattern of CIRI-associated TFs in the S3I3 and S24I24 datasets, and the hierarchical clustering method was selected as 'complete'.

\section{Results}

Differentially enriched GO terms associated with CIRI in tMCAO. Cerebral ischemia/reperfusion induces a cascade of secondary delayed cell deaths that are partially mediated by alterations in molecular transcriptional activities during reperfusion $(4,26)$. To improve understanding into the transcriptional mechanisms of CIRI, we performed a computational analysis workflow to identify candidate CIRI-associated TFs and their regulatory mechanisms, by combining a statistically principled GSEA with differentially expressed signature gene-sets post-analysis (Fig. 1).

GSEA of S3I3 and S24I24 datasets yielded a total of 423 and $953 \mathrm{GO}$ terms that were significantly enriched at 3 and $24 \mathrm{~h}$

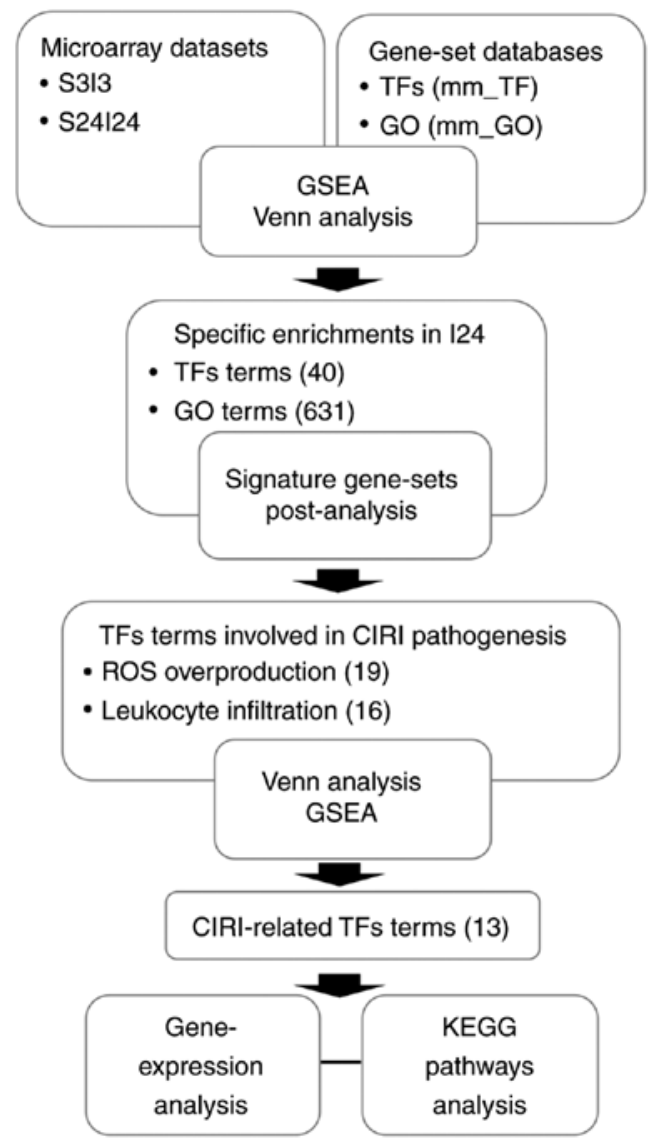

Figure 1. Workflow of analysis. The strategy was executed in a three-step manner. In the first step, gene set enrichment and Venn analyses were performed to identify the GO terms and TF-target gene-sets (TF terms) that were specifically enriched in the I 24 group. In the second step, we calculated the gene-sets similarity of TF-target gene-sets and GO terms obtained from the first step using the 'Post-Analysis' feature of EnrichmentMap and extracted the TF-target gene-sets associated with 'ROS biosynthesis' and 'leukocytes infiltration process'. These TFs were considered as CIRI-associated TFs. In the final step, the transcriptional expression patterns of these CIRI-related TFs and their functions involved in CIRI pathogenesis were identified. CIRI, cerebral ischemia-reperfusion injury; GO, Gene Ontology; KEGG, Kyoto Encyclopedia of Genes and Genomes; ROS, reactive oxygen species; TF, transcription factor.

after reperfusion in tMCAO mice, respectively (Table II). As CIRI is generally considered to peak at $\sim 24 \mathrm{~h}$ after reperfusion $(2,27)$, we further identified the GO terms specifically regulated $24 \mathrm{~h}$ after reperfusion by Venn analysis of the enriched GO terms between the I3 and I 24 groups. To improve understanding of the biological significance of these I24-specific GO terms, 631 GO terms were grouped into several major clusters with semantic annotations (Fig. 2), most of which were previously reported to be associated with the pathogenesis of CIRI, including 'lymphocytes mediated immunity' (55 terms), 'lymphocytes proliferation' (55 terms), 'nucleocytoplasmic transport' (24 terms), 'JAK-STAT cascade' (19 terms), 'Toll-like receptor pathway' (16 terms), 'reactive oxygen species (ROS) biosynthetic process' (13 terms), 'cytokine secretion' (12 terms), 'endopeptidase activity' (12 terms) and 'coagulation' (11 terms). A total of $631 \mathrm{GO}$ terms were specifically enriched $24 \mathrm{~h}$ following reperfusion; four terms were downregulated and 627 terms were upregulated (Fig. 3A). 
Table II. Number of gene-sets differentially enriched in tMCAO at $3 \mathrm{~h}$ (S3I3) and $24 \mathrm{~h}$ (S24I24) after reperfusion.

\begin{tabular}{lccccccrr}
\hline & \multicolumn{3}{c}{ S3I3 dataset } & & \multicolumn{3}{c}{ S24I24 dataset } \\
\cline { 2 - 3 } Gene-sets & Upregulated & Downregulated & Total & & Upregulated & Downregulated & Total \\
\hline Transcription factor-targets & 34 & 1 & 35 & & 65 & 0 & 65 \\
Gene ontology-terms & 423 & 0 & 423 & & 949 & 4 & 953
\end{tabular}

tMCAO. transient middle cerebral artery occlusion.

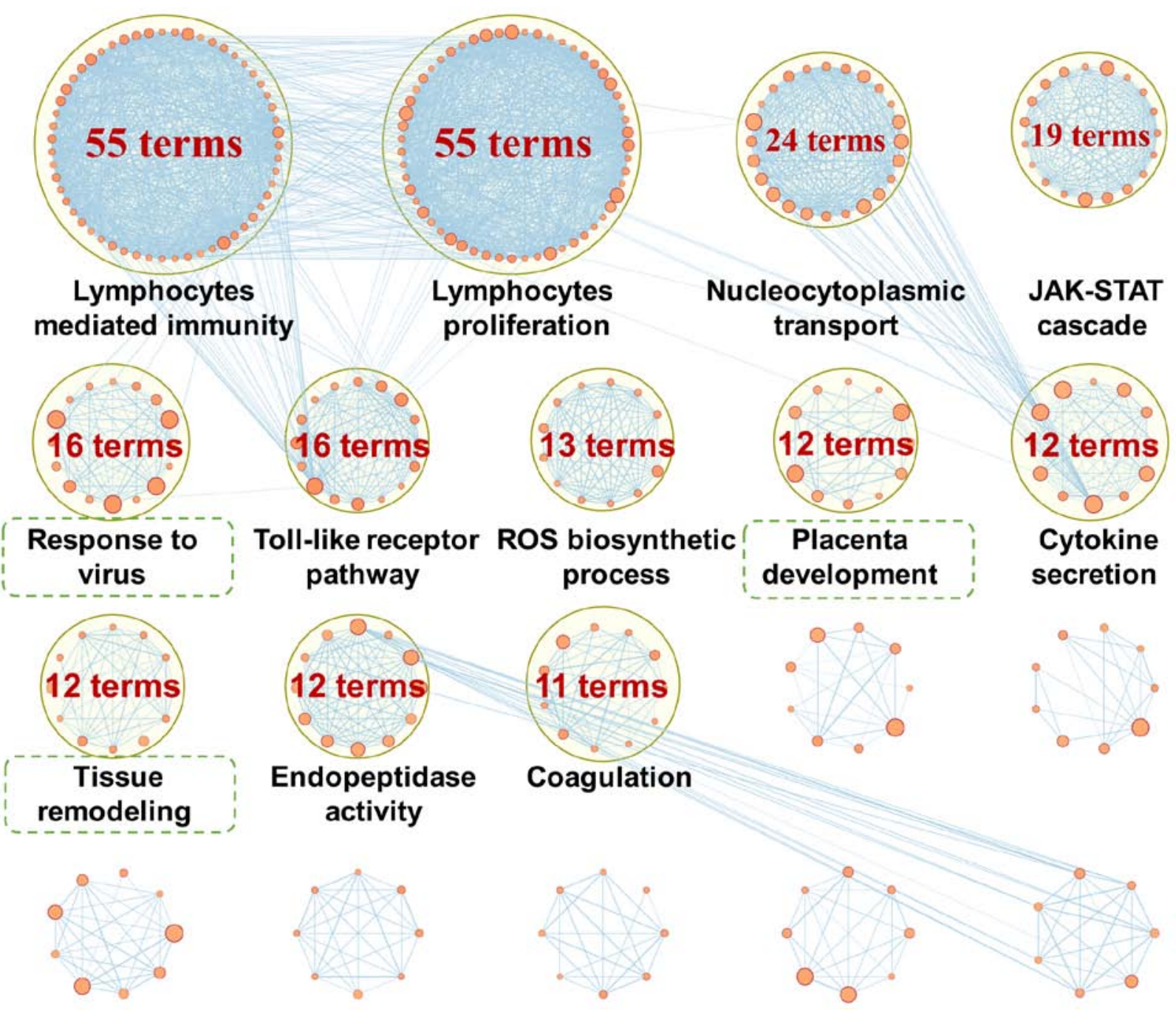

Figure 2. Clusters of functionally-associated I24-specific GO terms. The enrichment results of I24-specific biological pathways and processes were mapped to a network of GO terms (nodes), where the node size represents the number of genes within each GO term and the thickness of blue lines (edges) represents the degree of GO term similarity. The network was automatically clustered and circled by AutoAnnotate software; each cluster was textually annotated and visualized in the EnrichmentMap software. This clustered network map was manually curated removing general and uninformative sub-networks, resulting in a simplified network. The labels marked with dashed lines indicate the clusters that are functionally unassociated with the present study. GO, Gene Ontology; STAT, signal transduction and activator of transcription.

Identification of key transcription factors associated with CIRI. Oxidative stress and inflammation are the two most important mechanisms in the pathogenesis of CIRI, in which ROS overproduction and leukocyte infiltration are key initiation processes (1). Therefore, TFs involving both ROS generation and leukocytes infiltration processes are likely to participate in the pathogenesis of CIRI.
Following GSEA using mm_TF gene-sets database, we identified 35 and 65 TF-target gene-sets that were significantly enriched at 3 and $24 \mathrm{~h}$ after reperfusion in tMCAO mice, with $40 \mathrm{TF}$-target gene-sets specifically enriched at $24 \mathrm{~h}$ after reperfusion (I24-specific TF-target gene-sets, Table II and Fig. 3A). Then, the gene set similarity of I24-specific TF-target gene-sets to I24-specific GO terms 
Table III. General description of candidate cerebral ischemia-reperfusion injury-related TF-target gene-sets and their target leading edge genes.

\begin{tabular}{|c|c|c|c|c|}
\hline TF gene-sets & Symbol & Name & Description & Leading edge genes \\
\hline ZHAO_SP3 & sp3 & SP3 & $\begin{array}{l}\text { Trans-acting } \\
\text { transcription factor } 3\end{array}$ & $\begin{array}{l}\text { Tgm1/Col1a2/Ccl2/Mmp9/Ucp2/Cdkn1a/ } \\
\text { Rac2/Tal1/Fbln1/Pcyt1a/Abcc1/Aebp1 }\end{array}$ \\
\hline ZHAO_CEBPB & $c e b p b$ & $\mathrm{C} / \mathrm{EBP}$ beta & $\mathrm{C} / \mathrm{EBP} \beta$ & $\begin{array}{l}\text { Saa3/Il6/Abcb1b/Cdkn1a/C3 } \\
\text { /Nupr1/Col1a1 }\end{array}$ \\
\hline WINGENDER_RELA & rela & P65 & $\begin{array}{l}\text { v-rel reticuloendotheliosis } \\
\text { viral oncogene homolog } \\
\text { A (avian) }\end{array}$ & $\begin{array}{l}\text { Il6/Myd88/Tlr13/Tlr2/ } \\
\text { Ccnd1/Lcn2/Serpine1/Zfp36 }\end{array}$ \\
\hline WINGENDER_CEBPA & cebpa & C/EBP alpha & $\mathrm{C} / \mathrm{EBP} \alpha$ & $\begin{array}{l}\text { Il6/Fabp4/Hmox1/Lcn2/ } \\
\text { Serpine1/Cebpa/Abcc3/ }\end{array}$ \\
\hline TFACTS_TP53 & $\operatorname{trp53}$ & P53 & $\begin{array}{l}\text { Transformation related } \\
\text { protein } 53\end{array}$ & $\begin{array}{l}\text { Bcl3/Upp1/Igfbp3/Casp1/Spp1/Xpc/ } \\
\text { Eomes/Ccnd1/Brca1/Birc5/Ezh2/ } \\
\text { Cdkn1a/Myc/Mki67/C1s1/Trp53/Abcc3/ } \\
\text { Rela/Phlda3/Notch1/Bak1/Abcc1/ } \\
\text { Rrm1/Afp/Prkab1 }\end{array}$ \\
\hline TFACTS_TFAP2A & tfap $2 a$ & AP-2 alpha & Transcription factor AP-2 $\alpha$ & $\begin{array}{l}\text { Il6/Timp1/Igfbp3/Nos3/Mmp9/Hk2/Th/ } \\
\text { Hmox1/Pecam1/Gfap/Cdkn1a/Myc/ } \\
\text { Plaur/Hook2/Cebpa/Fbln1/Plat/Mcam/ } \\
\text { Adm/Calb2/Tnpo1/Col1a1/Taf7/Cyp11a1 }\end{array}$ \\
\hline TFACTS_STAT5B & stat $5 b$ & STAT5B & $\begin{array}{l}\text { Signal transducer and } \\
\text { activator of transcription } 5 \mathrm{~B}\end{array}$ & $\begin{array}{l}\text { Socs3/Myd88/Tlr2/Fcgr1/A2m/Cd84/ } \\
\text { Ccnd1/Klk8/Igf1/Cish }\end{array}$ \\
\hline TFACTS_STAT5A & stat $5 a$ & STAT5A & $\begin{array}{l}\text { Signal transducer and } \\
\text { activator of transcription } 5 \mathrm{~A}\end{array}$ & $\begin{array}{l}\text { Socs3/Myd88/Tlr2/Fcgr1/A2m/ } \\
\text { Cd84/Ccnd1/Klk8/Igf1/Cish }\end{array}$ \\
\hline TFACTS_SPI1 & spil & PU.1 & $\begin{array}{l}\text { Spleen focus forming } \\
\text { virus proviral integration } \\
\text { oncogene }\end{array}$ & $\begin{array}{l}\text { Ncf4/Vav1/Fcgr1/Cd163/Itgb2/ } \\
\text { Nos3/Ptprc/Prtn3/Tlr4/Msr1/Cd68/Cd72/ } \\
\text { Ctsk/Tal1/Pdgfrb/Il1b/Chil1/Lsp1/ } \\
\text { Icam1/Fli1 }\end{array}$ \\
\hline TFACTS_GLI2 & gli2 & GLI2 & $\begin{array}{l}\text { GLI-Kruppel family member } \\
\text { GLI2 }\end{array}$ & $\begin{array}{l}\text { Il6/Tnc/Tgfbi/Il1r2/Igfbp3/Rapgefl1/ } \\
\text { Tead1/Ccnd1/Ccl17/Irf9/Fen1/Bmp7/ } \\
\text { Cdkn1a/Myc/Hpgd/Npy2r/Efna1/ } \\
\text { Ifngr1/Itga6/Lum/Cks1b/Cflar/ } \\
\text { Col5a2/Edn1/Lamb3/Mad211 }\end{array}$ \\
\hline TFACTS_FOS & fos & FOS & FBJ osteosarcoma oncogene & $\begin{array}{l}\text { Mmp3/Il6/Tgm1/Itga5/Timp1/ } \\
\text { Th/Msr1/Plaur/Nqo1/Trp53 }\end{array}$ \\
\hline TFACTS_EGR1 & egrl & EGR1 & Early growth response 1 & $\begin{array}{l}\text { Il6/Cd44/Col1a2/Spint1/Apoa1/Cebpb/Th/ } \\
\text { Hmox1/Nudt6/Tfpi2/Pecam1/Twist1/ } \\
\text { Serpine1/Trp53/Elk1/Cebpa/ } \\
\text { Rela/Cd9/Icam1 }\end{array}$ \\
\hline TFACTS_JUND & jund & JUND & Jun D proto-oncogene & $\begin{array}{l}\text { Il6/Fos11/Timp1/Spp1/Th/Cdkn1a/Plaur/ } \\
\text { Nqo1/Plat }\end{array}$ \\
\hline
\end{tabular}

C/EBP, CCAAT/enhancer binding protein; TF, transcription, factor.

were determined using the 'Post-Analysis' feature of EnrichmentMap; 19 and 16 TF-target gene-sets were significantly associated with 'ROS biosynthetic process' and 'leukocytes infiltration', respectively (Fig. 3B). Of these TF-target gene-sets, 15 were finally determined as candidate CIRI-related TF-target gene-sets that were associated with 'ROS biosynthesis' and 'leukocyte infiltration'. In addition, we further employed these 15 CIRI-related
TF-target gene-sets in the I3I24 dataset for GSEA to identify CIRI-related TFs that were significantly induced after $24 \mathrm{~h}$ of reperfusion than after $3 \mathrm{~h}$. From the GSEA results, two TFs, upstream transcription factor 1 (Usfl) and Cebpd were excluded as they were not significantly enriched in the I24 group under the cutoff adjusted $\mathrm{P} \leq 0.05$ (Fig. 3C). An overview of these 13 candidate CIRI-associated TF-target gene-sets is presented in Table III. 
A

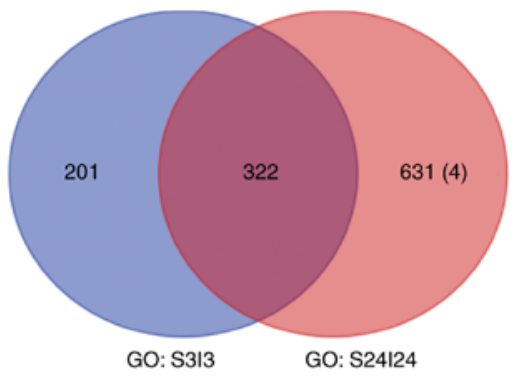

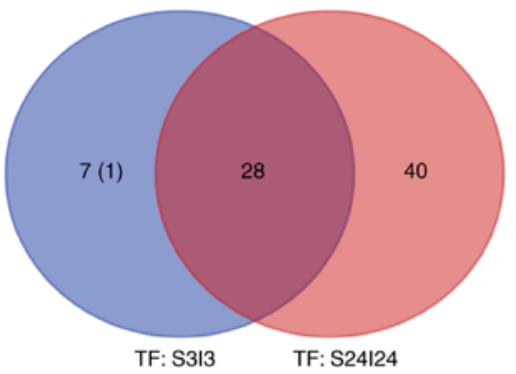

B

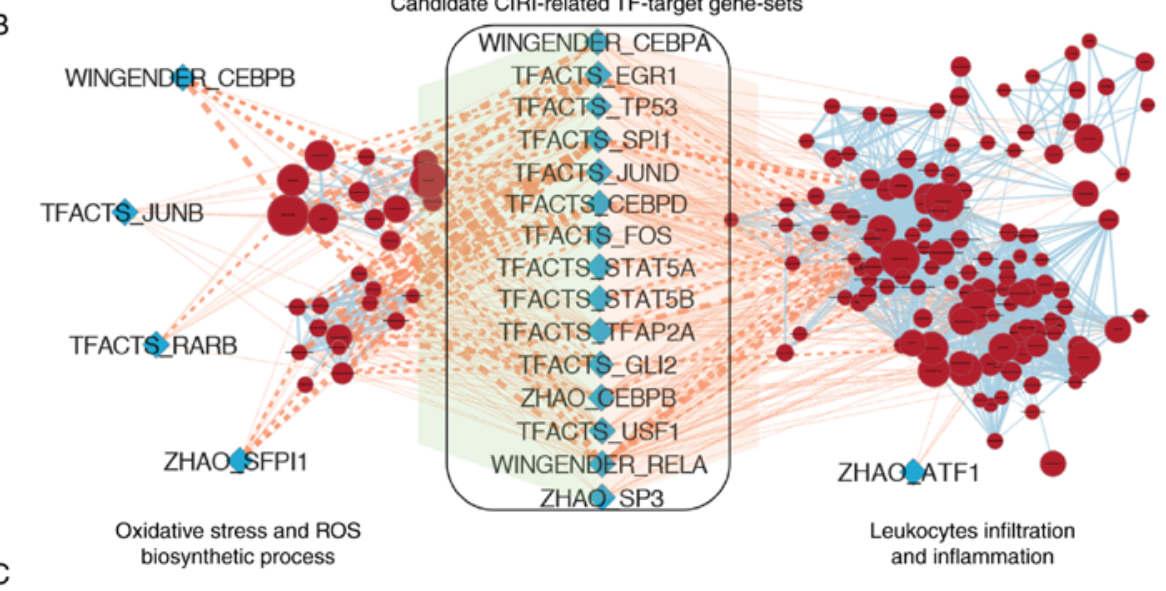

C

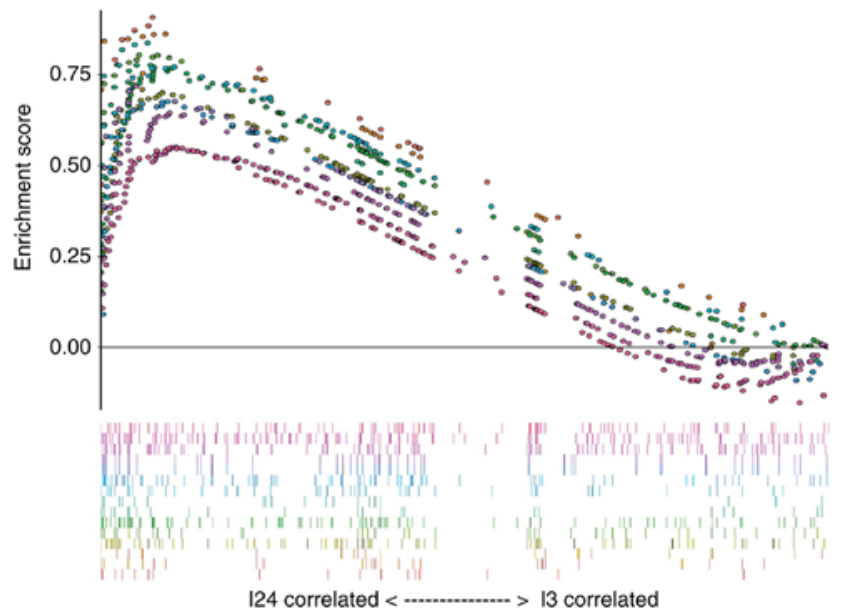

and inflammation

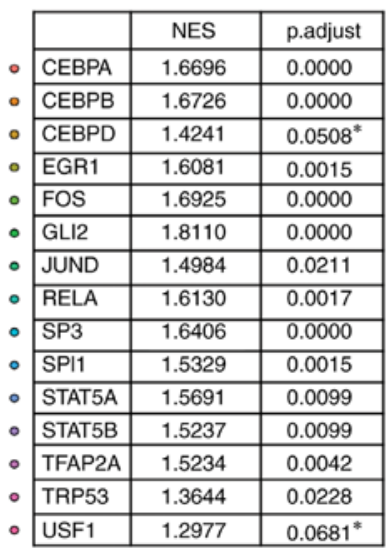

Figure 3. Identification of key transcription factors associated with CIRI. (A) Venn diagrams of the numbers of GO terms (left panel) or TF-target gene-sets (right panel) significantly enriched in the ischemic brains of middle cerebral artery occlusion mice after $3 \mathrm{~h}$ (S3I3) or 24 h (S24I24) of reperfusion. The number of terms enriched in the sham control group was shown in parentheses. (B) Enrichment map for CIRI-associated GO terms and overlap with TF-target gene-sets. The map presented the specifically enriched GO terms of 'ROS biosynthesis' and 'leukocytes infiltration' process (brick red circles) in I24 group vs. sham controls. The blue diamonds represented the TF-target gene-sets that were specifically enriched in the I24 group. Pink edges indicated overlap between the TF-target gene-sets and enriched GO terms; thickness represented significance. Only edges with nominal $\mathrm{P}<0.05$ based on a Mann-Whitney U test were visualized. (C) Identification of CIRI-related TF-target gene-sets by gene set enrichment analysis between I3 and I24 groups. A total of two TFs, Usfl, and Cebpd, with adjusted P>0.05, were marked with asterisks and excluded for the further study. CEBP, CCAAT enhancer binding protein; CIRI, cerebral ischemia-reperfusion injury; GO, gene ontology; ROS, reactive oxygen species; STAT, signal transduction and activator of transcription; TF, transcription factor; TRP53, transformation related protein 53; Usf1, upstream transcription factor 1.

Gene expression analysis of CIRI-related TFs and their target genes. Although all the CIRI-related TF-target gene-sets were specifically induced in the injured brain after $24 \mathrm{~h}$ of reperfusion, gene expression analysis demonstrated that these CIRI-related TFs and their target genes exhibited variable transcriptional expression patterns in S3I3 or S24I24 dataset. As presented in Fig. 4A, one group of TFs including Stat $5 b$, transformation related protein 53 (Trp53), TF AP-2 $\alpha$ (Tfap2a), spleen focus forming virus proviral integration oncogene (Spil), and Sp3 exhibited no significant difference in expression in the S3I3 and
S24I24 datasets, while another group of TFs with Fos and Egrl were significantly upregulated in both datasets. GLI family zinc finger 2 (Gli2) and Jund exhibited opposing expression patterns, in which Gli2 was downregulated in the S24I24 dataset while Jund was upregulated in the S3I3 dataset. The last group of TFs with Stat $5 a$, Rela, Cebpb and Cebpa were overexpressed specifically in the S24I24 dataset but not in S3I3.

In addition, we performed leading-edge analysis with the 13 CIRI-related TF-target gene-sets following GSEA between the I 3 and I 24 groups to identify the target genes regulated by 
A

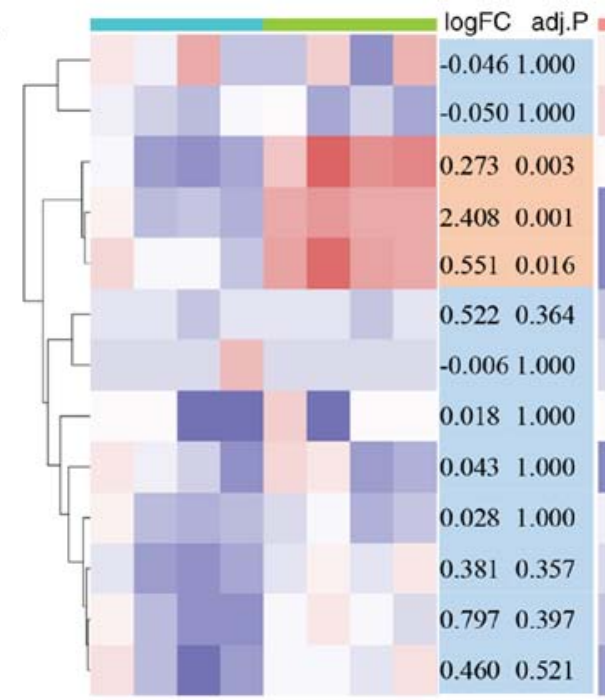

$\log \mathrm{FC}$ adj.P

\begin{tabular}{|c|c|c|c|}
\hline-0.122 & 0.804 & Stat5b & Groups \\
\hline-0.396 & 0.012 & Gli2 & 13 \\
\hline 0.071 & 0.312 & Jund & $\begin{array}{l}\mathrm{S} 24 \\
\mathrm{~S} 3\end{array}$ \\
\hline 2.589 & 0.000 & Fos & ${ }^{0}$ adj.P.Val \\
\hline 0.419 & 0.020 & Egr1 & $\begin{array}{ll}-1 & <0.05\end{array}$ \\
\hline .079 & 0.138 & Trp53 & -2 \\
\hline .028 & 0.180 & Tfap2a & \\
\hline 0.022 & 0.717 & Spi1 & \\
\hline .484 & 0.210 & Sp3 & \\
\hline 1.500 & 0.001 & Stat5a & \\
\hline 0.822 & 0.006 & Rela & \\
\hline 2.504 & 0.001 & Cebpb & \\
\hline 1.282 & 0.005 & Cebpa & \\
\hline
\end{tabular}

B

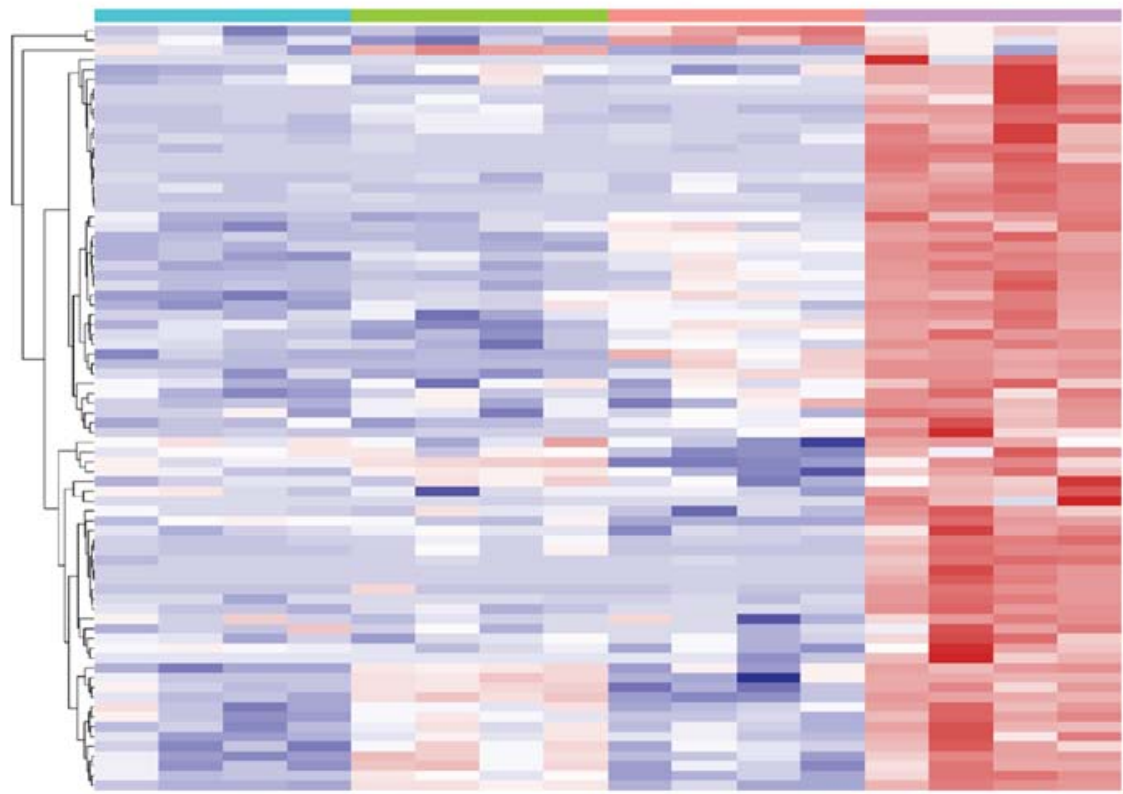

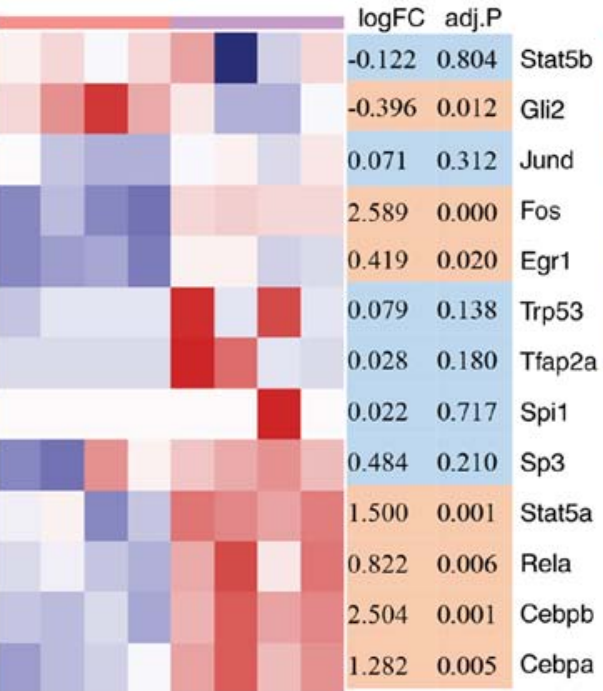

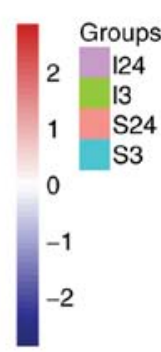

Figure 4. Heatmap analysis of CIRI-related TFs and their target genes. (A) The transcriptional expression patterns of CIRI-related TF genes in the ischemic brain after 3 and $24 \mathrm{~h}$ of reperfusion compared with the relevant sham controls. The color blocks in the lower right of heatmap represent the significance of the differential expression of CIRI-related TF genes, orange, adjusted $\mathrm{P}<0.05$; light blue, adjusted $\mathrm{P}>0.05$. (B) Heat map of CIRI-related TFs target genes $(\mathrm{n}=78)$ significantly upregulated (adjusted $\mathrm{P}<0.05)$ in the I24 group as compared with sham control subjects (S24). These genes were identified by leading-edge analysis after gene set enrichment analysis between I 3 and I24 groups, and the $\log 2$ transformed data were used for heatmap analysis. The y-axis data are individual genes, the color bar in the heatmap right represents the gene expression level (blue, downregulation and red, upregulation). The color blocks in the upper right of the heatmap indicate the experimental groups (purple, I24 group; green, I3 group; pink, S24 group and cyan, S3 group). CEBP, CCAAT enhancer binding protein; CIRI, cerebral ischemia-reperfusion injury; Egrl, early growth response-1; Gli2, GLI family zinc finger 2; Spi1, spleen focus forming virus proviral integration oncogene; Stat, signal transduction and activator of transcription; TF, transcription factor.

these CIRI-related TFs. A total of 123 target genes regulated by the 13 CIRI-associated TFs were identified in the I3I24 dataset, of which 78 genes were significantly overexpressed in the I24 group and were presented as a heatmap (Fig. 4B). The statistical results of expression of the 78 target genes in the S3I3 and S24I24 datasets were listed in Table S1.

Enrichment analysis for target genes of CIRI-associated transcription factors. The 78 overexpressed genes in the I 24 group were used for enrichment analysis to determine significant KEGG pathways in which CIRI-related TFs were involved. The results demonstrated that these target genes of CIRI-related TFs were mainly involved in the 'PI3K-Akt signaling pathway' (adjusted $\mathrm{P}=3.54 \times 10^{-5}$ ), 'tumor necrosis factor (TNF) signaling pathway' (adjusted $\mathrm{P}=4.01 \times 10^{-7}$ ), 'hypoxia-inducible factor-1 (HIF-1) signaling pathway' (adjusted $\mathrm{P}=3.81 \times 10^{-7}$ ), 'focal adhesion' (adjusted $\mathrm{P}=1.55 \times 10^{-4}$ ) and 'JAK-STAT signaling pathway' (adjusted $\mathrm{P}=5.74 \times 10^{-5}$ ) (Fig. $5 \mathrm{~A}$ ). In addition, the KEGG pathways involving each CIRI-related TF were further analyzed by calculating the gene-sets similarity of CIRI-related TFs with the KEGG pathways enriched in the S24I24 dataset using the 'Post-Analysis' feature of EnrichmentMap (Fig. 5B and $\mathrm{C}, \mathrm{P} \leq 0.05)$. Of these CIRI-related TFs, 5 TFs (Cebpa, Gli2, Sp3, Tfap2a and Spil) were reported to be associated with CIRI, which were mainly involved in the pathways related to inflammation and responses to reperfusion, including the 
A

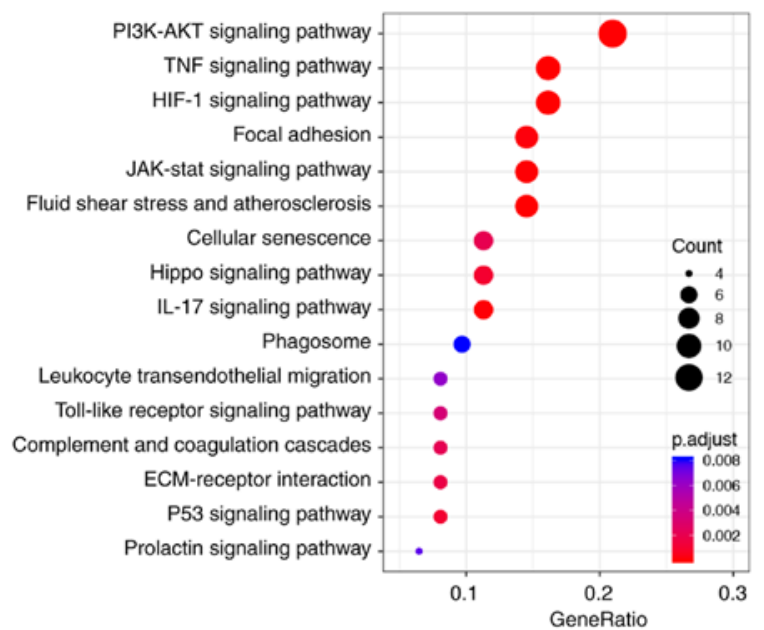

B

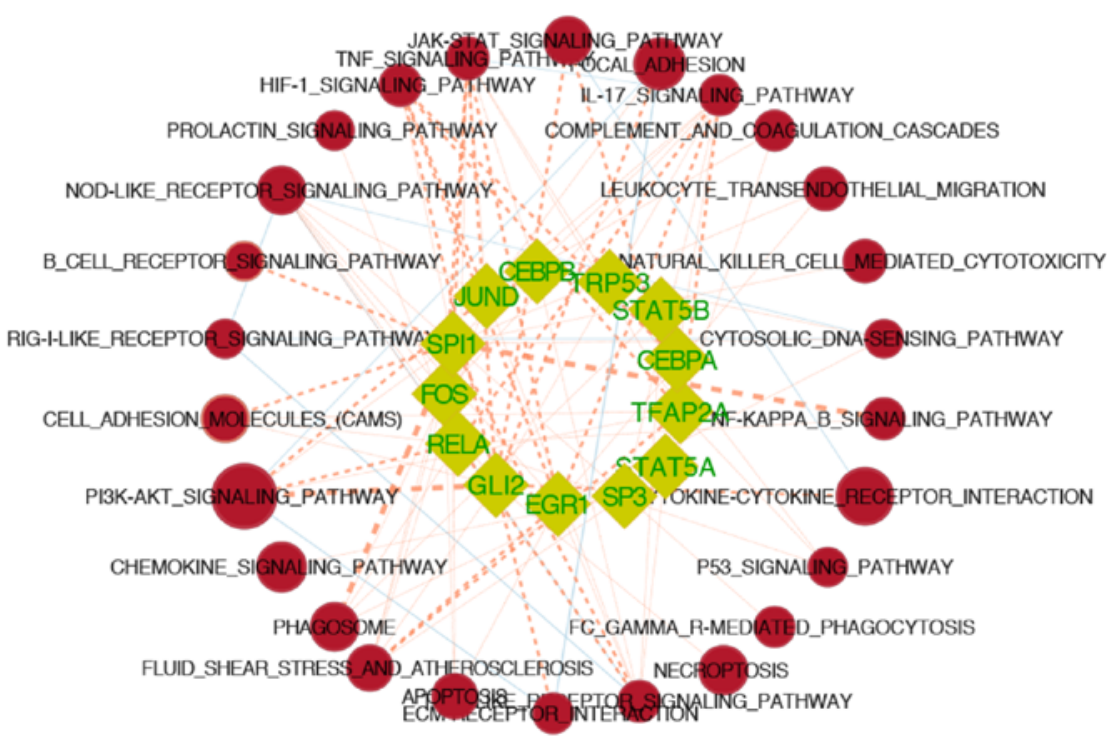

C

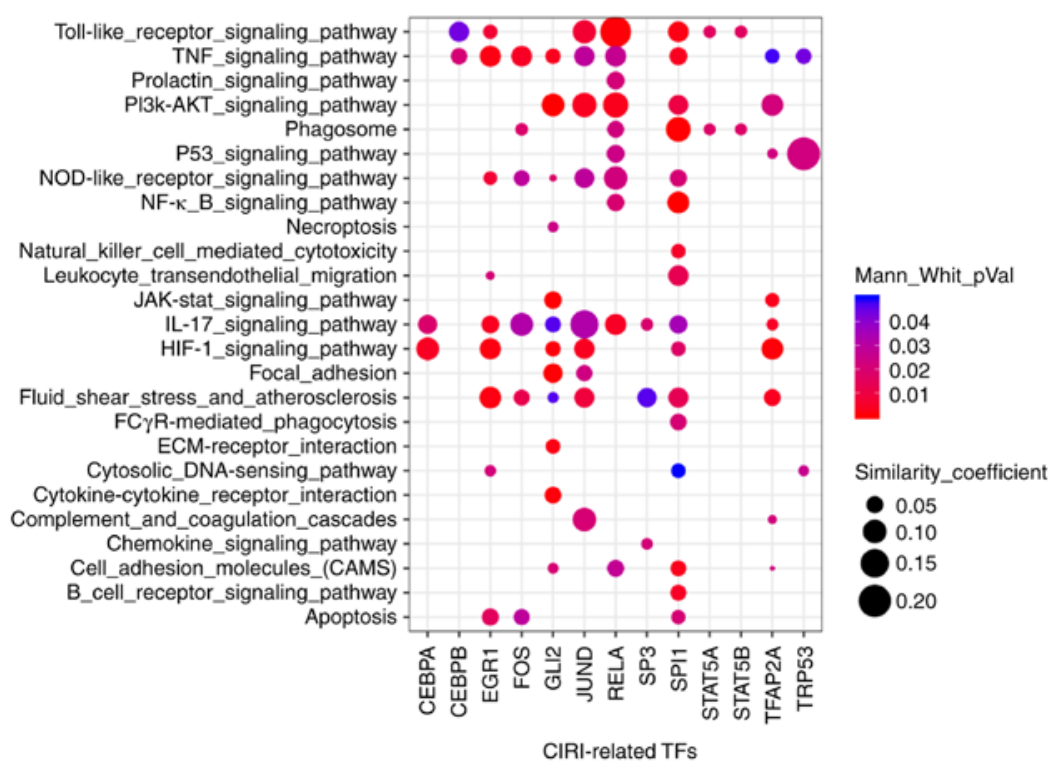

Figure 5. Functional enrichment analysis of CIRI-associated TFs and their target genes. (A) Dot plot showing enrichment of KEGG pathway for the list of CIRI-related TFs target genes (adjusted P<0.05). The dot sizes represent the numbers of genes, the color of dots corresponds to the adjusted P-value. (B) Enrichment map for KEGG pathways and overlap with TF-target gene-sets. The map indicated the specifically enriched KEGG pathways (brick red filled circles) in I24 group vs. sham controls. The yellow diamonds represented the TF-target gene-sets that were specifically enriched in the I24 group. Pink edges indicated overlap between the TF-target gene-sets and enriched KEGG pathways; thickness represented significance. Only edges with nominal $\mathrm{P}<0.05$ based on a Mann-Whitney U test were visualized. (C) Dot plot of KEGG pathway enrichment for each CIRI-related TF. The dot sizes represent the coefficient similarity of the KEGG pathway and CIRI-related TF; the color of dots corresponds to the Mann-Whitney U test nominal P-value. CIRI, cerebral ischemia-reperfusion injury; IL, interleukin; KEGG, Kyoto Encyclopedia of Genes and Genomes; PI3K, phosphatidylinositol 3-kinase; TF, transcription factor. 
'TNF signaling pathway' (Gli2, Spil and Tfap2a, $\mathrm{P}=0.0035$, 0.0035 and 0.048 , respectively), 'interleukin (IL)-17 signaling pathway' (Cebpa, Gli2, Sp3, Spil and Tfap2a, $\mathrm{P}=0.019,0.047$, $0.019,0.035$ and 0.005 , respectively), and fluid shear stress and atherosclerosis (Gli2, Sp3, Spil and Tfap2a, $\mathrm{P}=0.047,0.046$, 0.013 and 0.003 , respectively). In particular, Spil was observed to be significantly involved in the majority of the enriched KEGG pathways of S24I24, including 'NF-kB signaling pathway' $(\mathrm{P}<0.001)$, 'phagosome' $(\mathrm{P}<0.001)$, 'Toll-like receptor signaling pathway' $(\mathrm{P}=0.002)$, 'TNF signaling pathway' ( $\mathrm{P}=0.004)$, 'PI3K-Akt signaling pathway' $(\mathrm{P}=0.010)$, 'HIF-1 signaling pathway' $(\mathrm{P}=0.017)$, 'apoptosis' $(\mathrm{P}=0.021)$, 'cell adhesion molecules' $(\mathrm{P}=0.003)$, 'NOD-like receptor signaling pathway' $(\mathrm{P}=0.021)$, 'cytosolic DNA-sensing pathway' $(\mathrm{P}=0.050)$, 'natural killer cell mediated cytotoxicity' $(\mathrm{P}=0.005)$, 'IL-17 signaling pathway' $(\mathrm{P}=0.035)$, 'B cell receptor signaling pathway' ( $\mathrm{P}=0.004)$, 'FC $\gamma \mathrm{R}$-mediated phagocytosis' $(\mathrm{P}=0.021)$, 'leukocyte trans-endothelial migration' $(\mathrm{P}=0.013)$, and 'fluid shear stress and atherosclerosis' $(\mathrm{P}=0.013)$.

\section{Discussion}

In the present study, the gene expression profiles of ischemic stroke were systematically analyzed at different time points ( 3 and $24 \mathrm{~h}$ ) after reperfusion using the GSEA bioinformatics method to reveal the possible transcriptional regulatory mechanisms associated with CIRI at various functional levels. We identified 13 key CIRI-related TFs, of which 5 CIRI-related TFs (Cebpa, Gli2, Sp3, Tfap2a and Spil) were reported, and the biological pathways involved following ischemic stroke were determined. To the best of our knowledge, this is the first bioinformatics study to characterize CIRI-related TFs in the context of the pathogenesis of CIRI.

Oh et al (28) investigated genetic alterations in the peripheral blood after acute ischemic stroke, suggesting the detrimental effects of peripheral white blood cells on the ischemic brain after reperfusion. In the present study, the GO terms specifically enriched at $24 \mathrm{~h}$ post-reperfusion in an MCAO rat model were analyzed to determine the CIRI-related TFs associated with ROS overproduction and leukocyte infiltration. It has been reported that differentially expressed genes at $24 \mathrm{~h}$ after cerebral ischemia/reperfusion in MCAO rats are associated with inflammation, apoptosis, stress and immune responses, and are involved in the pathogenesis of CIRI $(29,30)$. Our results from GSEA are consistent with previous findings, indicating that I24-specific GO terms were mainly enriched in immune cells activity and inflammation, and their related pathways, including the Jak-stat cascade and the Toll-like receptor pathway. In addition, GSEA also revealed several functional clusters of CIRI-related GO terms that have not previously been reported in high throughput studies (30), such as 'nucleocytoplasmic transport,' 'endopeptidase activity' and 'coagulation'. Importantly, the enrichment of nucleocytoplasmic transport-associated genes could reveal the enhancement of transcriptional activity in the ischemic brain $24 \mathrm{~h}$ after reperfusion, further indicating the pivotal roles of novel transcripts induced by reperfusion in the pathogenesis of CIRI.

Transcription factors can be induced in ischemic stroke, and are involved in neuronal injury or neuroprotection at different stages after reperfusion by regulating post-ischemic inflammation (6). In the present study, it was determined that 8 CIRI-related TFs, including Cebpb, Egrl, Rela, Jund, Stat5a/b, Fos and $\operatorname{Trp} 53$ were functionally activated at $24 \mathrm{~h}$ after reperfusion, as other studies have previously reported $(6,31,32)$. Of these TFs, the activation of Jund (31), Stat5a/b (32), Fos and Trp53 is known to prevent ischemic neuronal damage, whereas the induction of Cebpb, Egrl and Rela promotes inflammation and neuronal death following cerebral ischemia (6). KEGG pathway analysis revealed that these CIRI-related TFs were not only involved in pro-inflammatory pathways to promote cell death but in neuroprotective pathways to promote neuron survival. These results demonstrated that TFs were important endogenous modulators, which may be actively involved in the balance of brain damage and repair during the pathogenesis of CIRI, by coordinating numerous downstream genes that govern inflammation.

Furthermore, the present study reported that three CIRI-related TFs (Jund, Fos and Egrl), belonging to the immediate early gene family, were significantly upregulated as early as $3 \mathrm{~h}$ after reperfusion. These TFs could be rapidly induced at the onset of hypoxic conditions to trigger ( $E g r l)$ or suppress (Jund and Fos) the first wave of inflammatory responses in the ischemic brain or other organs $(11,33)$. An additional group of CIRI-related TFs, including Stat5a, Rela, and Cebpa/b, can be induced by Fos and Egrl, and were significantly overexpressed $24 \mathrm{~h}$ after reperfusion. These TFs may act in a positive regulatory loop and promote of CIRI at $24 \mathrm{~h}$ after reperfusion (34).

Other CIRI-related TFs, including Cebpa, Gli2, Sp3, Tfap $2 a$ and Spil were first identified to be associated with CIRI in this study. The TFs Cebpa and Spil are two myeloid-specific proteins involved in the differentiation of monocyte lineages (35). A previous study revealed that the decrease in CEBP $\alpha / \beta$ DNA-binding activity was inevitably associated with CIRI in a long-term (30 days) global cerebral ischemia model (36). In addition, the expression of Cebpa was induced in microglia after hypoxic-ischemic brain injury (37). In addition, Cebpa can combine with Spil to enhance monocyte-specific promoter activity and affect NF- $\mathrm{KB}$ activity in response to TNF- $\alpha$ signaling (38). It is well reported that increased inflammation and inflammatory proteins, such as TNF- $\alpha$, serve critical roles in the pathogenesis of CIRI via NF-кB signaling pathway (34). Therefore, Spil and Cebpa may serve a synergistic pro-inflammatory role in the pathogenesis of CIRI. Sp3 is a transcriptional repressor that, in complex with RE1-silencing transcription factor, inhibits the gene expression of $\mathrm{Na}^{+}-\mathrm{Ca}^{2+}$ exchanger 1 in the tMCAO rat model (39). Our functional enrichment analysis demonstrated that $S p 3$ was activated in the I24 group and was functionally associated with the activation of the IL-17 signaling pathway. Previous studies have reported that inhibiting the IL-17 signaling pathway reduced neutrophil infiltration and protected against ischemic stroke (40). Sp3 activation may promote neutrophil invasion via the IL-17 signaling pathway, which then promotes the pathogenesis of CIRI-induced cerebral edema.

Gli2 is generally considered as a potent oncogene by activating the Sonic hedgehog (Shh) signaling pathway in embryonal cancer cells $(41,42)$. Previously, the Shh signaling pathway was shown to serve an important role in neuroprotection and neurogenesis via a mechanism of anti-oxidative stress 
in a rodent stroke model after cerebral ischemia (43). In the present study, the transcriptional activity of Gli2 was significantly activated and closely associated with the PI3K-Akt signaling pathway, suggesting the neuroprotective effect of Gli2 in response to CIRI. Tfap $2 a$ has been considered to be a tumor-related gene that regulates tumor growth and survival via the HIF-1 $\alpha$ signaling pathway (44). It was previously demonstrated that Tfap $2 a$ served an important role in the repair of myocardial ischemic injury via an Akt-dependent signaling pathway (45). The present study reported that Tfap $2 a$ activation was involved in the HIF-1 and PI3K-Akt signaling pathways, suggesting other biological functions of Tfap $2 a$ in the pathogenesis of CIRI in addition to tumorigenesis. Collectively, these findings indicated that these CIRI-related TFs served complex and critical roles in the pathogenesis of CIRI at the level of various pathways following ischemia/reperfusion. This supports the hypothesis that TFs could be effective targets for alleviating CIRI.

The lack of further experimental verification of these CIRI-TFs is the largest limitation of the present study. In particular, the newly identified CIRI-associated TFs, including Gli2, Spil and Tfap $2 a$ require further investigation to determine their protein expression profiles and exact roles in the pathogenesis of CIRI.

In summary, the functional gene-sets enriched in ischemic stroke at different time points ( 3 and $24 \mathrm{~h}$ ) after reperfusion based on microarray expression data were analyzed in the present study. The results indicated 13 CIRI-related TFs, including Cebpb, Cebpa, Egrl, Fos, Rela, Jund, Stat5a/b, Trp53, Gli2, Sp3, Tfap2a and Spil, which may serve significant regulatory roles in the pathogenesis of CIRI. Although some of these TFs were reported in previous studies, five TFs including Cebpa, Gli2, Sp3, Tfap2a and Spil have not been reported in CIRI to the best of our knowledge. These findings may provide insight into the potential molecular mechanism underlying the pathogenesis of CIRI and aid the identification of novel targets in the treatment of CIRI; however, further investigation is required.

\section{Acknowledgements}

Not applicable.

\section{Funding}

The present study was supported by the National Natural Science Foundation of China (grant nos. 81471767 and 81871464).

\section{Availability of data and materials}

The microarray data used during the current study can be downloaded from the GEO database (https://www.ncbi. nlm.nih.gov/geo/query/acc.cgi?acc=GSE32529). The mouse gene-sets of transcription factor target genes (mm_TF), Gene Ontology (mm_GO) and metabolic pathways (mm_metabolic) used in the present study can be downloaded from GSKB database (http://ge-lab.org/\#/data). The datasets used and/or analyzed during the current study are available from the corresponding author on reasonable request.

\section{Authors' contributions}

YYZ, JL and WJJ made substantial contributions to the design of the present study. YEL, KW and AFL conducted data collection and preprocessing. Data analysis and interpretation was performed by YYZ, KW, WW, YEL, JZ, CL, YQZ and APZ. YYZ and JL wrote the manuscript. All authors read and approved the final manuscript.

\section{Ethics approval and consent to participate}

Not applicable.

\section{Patient consent for publication}

Not applicable.

\section{Competing interests}

The authors declare that they have no competing interests.

\section{References}

1. $\mathrm{L} \mathrm{L}, \mathrm{X} \mathrm{W}$ and $\mathrm{Z} \mathrm{Y}$ : Ischemia-reperfusion injury in the brain: Mechanisms and potential therapeutic strategies. Biochem Pharmacol (Los Angel) 5: 213, 2016.

2. Hossmann KA: The two pathophysiologies of focal brain ischemia: Implications for translational stroke research. J Cereb Blood Flow Metab 32: 1310-1316, 2012.

3. Eltzschig HK and Eckle T: Ischemia and reperfusion-from mechanism to translation. Nat Med 17: 1391-1401, 2011.

4. VanGilder RL, Huber JD, Rosen CL and Barr TL: The transcriptome of cerebral ischemia. Brain Res Bull 88: 313-319, 2012.

5. Lu XC, Williams AJ, Yao C, Berti R, Hartings JA, Whipple R, Vahey MT, Polavarapu RG, Woller KL, Tortella FC and Dave JR: Microarray analysis of acute and delayed gene expression profile in rats after focal ischemic brain injury and reperfusion. J Neurosci Res 77: 843-857, 2004.

6. Yi JH, Park SW, Kapadia R and Vemuganti R: Role of transcription factors in mediating post-ischemic cerebral inflammation and brain damage. Neurochem Int 50: 1014-1027, 2007.

7. Cox-Limpens KE, Gavilanes AW, Zimmermann LJ and Vles JS: Endogenous brain protection: What the cerebral transcriptome teaches us. Brain Res 1564: 85-100, 2014.

8. Sun Y, Liu L, Yuan J, Sun Q, Wang N and Wang Y: RP105 protects PC12 cells from oxygenglucose deprivation/reoxygenation injury via activation of the PI3K/AKT signaling pathway. Int J Mol Med 41: 3081-3089, 2018.

9. Iadecola C, Salkowski CA, Zhang F, Aber T, Nagayama M, Vogel SN and Ross ME: The transcription factor interferon regulatory factor 1 is expressed after cerebral ischemia and contributes to ischemic brain injury. J Exp Med 189: 719-727, 1999.

10. Nurmi A, Lindsberg PJ, Koistinaho M, Zhang W, Juettler E, Karjalainen-Lindsberg ML, Weih F, Frank N, Schwaninger M and Koistinaho J: Nuclear factor-kappaB contributes to infarction after permanent focal ischemia. Stroke 35: 987-991, 2004.

11. Tureyen K, Brooks N, Bowen K, Svaren J and Vemuganti R: Transcription factor early growth response-1 induction mediates inflammatory gene expression and brain damage following transient focal ischemia. J Neurochem 105: 1313-1324, 2008.

12. Kapadia R, Tureyen K, Bowen KK, Kalluri H, Johnson PF and Vemuganti R: Decreased brain damage and curtailed inflammation in transcription factor CCAAT/enhancer binding protein beta knockout mice following transient focal cerebral ischemia. J Neurochem 98: 1718-1731, 2006.

13. Satriotomo I, Bowen KK and Vemuganti R: JAK2 and STAT3 activation contributes to neuronal damage following transient focal cerebral ischemia. J Neurochem 98: 1353-1368, 2006.

14. Ridder DA, Bulashevska S, Chaitanya GV, Babu PP, Brors B, Eils R, Schneider A and Schwaninger M: Discovery of transcriptional programs in cerebral ischemia by in silico promoter analysis. Brain Res 1272: 3-13, 2009. 
15. Pulliam JV, Xu Z, Ford GD, Liu C, Li Y, Stovall KC, Cannon VS, Tewolde T, Moreno CS and Ford BD: Computational identification of conserved transcription factor binding sites upstream of genes induced in rat brain by transient focal ischemic stroke. Brain Res 1495: 76-85, 2013.

16. Camos S, Gubern C, Sobrado M, Rodríguez R, Romera VG, Moro MA, Lizasoain I, Serena J, Mallolas J and Castellanos M: The high-mobility group I-Y transcription factor is involved in cerebral ischemia and modulates the expression of angiogenic proteins. Neuroscience 269: 112-130, 2014.

17. Subramanian A, Tamayo P, Mootha VK, Mukherjee S, Ebert BL, Gillette MA, Paulovich A, Pomeroy SL, Golub TR, Lander ES and Mesirov JP: Gene set enrichment analysis: A knowledge-based approach for interpreting genome-wide expression profiles. Proc Natl Acad Sci USA 102: 15545-15550, 2005.

18. Merico D, Isserlin R, Stueker O, Emili A and Bader GD Enrichment map: A network-based method for gene-set enrichment visualization and interpretation. PLoS One 5: e13984, 2010.

19. Stevens SL, Leung PY, Vartanian KB, Gopalan B, Yang T, Simon RP and Stenzel-Poore MP: Multiple preconditioning paradigms converge on interferon regulatory factor-dependent signaling to promote tolerance to ischemic brain injury. J Neurosci 31: 8456-8463, 2011.

20. Vartanian KB, Stevens SL, Marsh BJ, Williams-Karnesky R, Lessov NS and Stenzel-Poore MP: LPS preconditioning redirects TLR signaling following stroke: TRIF-IRF3 plays a seminal role in mediating tolerance to ischemic injury. J Neuroinflammation 8 : 140, 2011.

21. Gautier L, Cope L, Bolstad BM and Irizarry RA: Affy-analysis of Affymetrix GeneChip data at the probe level. Bioinformatics 20 : 307-315, 2004

22. Kucera M, Isserlin R, Arkhangorodsky A and Bader GD: AutoAnnotate: A cytoscape app for summarizing networks with semantic annotations. F1000Res 5: 1717, 2016.

23. Yu G, Wang LG, Han Y and He QY: ClusterProfiler: An R package for comparing biological themes among gene clusters. OMICS 16: 284-287, 2012.

24. Wickham H: Ggplot2: Elegant graphics for data analysis Springer, New York, Statistics and Computing/Statistics Programs VIII, 213, 2009.

25. Ritchie ME, Phipson B, Wu D, Hu Y, Law CW, Shi W and Smyth GK: limma powers differential expression analyses for RNA-sequencing and microarray studies. Nucleic Acids Res 43: e47, 2015 .

26. Kim JB, Piao CS, Lee KW, Han PL, Ahn JI, Lee YS and Lee JK Delayed genomic responses to transient middle cerebral artery occlusion in the rat. J Neurochem 89: 1271-1282, 2004.

27. Zhang RL, Chopp M, Chen H and Garcia JH: Temporal profile of ischemic tissue damage, neutrophil response, and vascular plugging following permanent and transient $(2 \mathrm{H})$ middle cerebral artery occlusion in the rat. J Neurol Sci 125: 3-10, 1994

28. Oh SH, Kim OJ, Shin DA, Song J, Yoo H, Kim YK and Kim JK Alteration of immunologic responses on peripheral blood in the acute phase of ischemic stroke: Blood genomic profiling study. J Neuroimmunol 249: 60-65, 2012.

29. Shao X, Bao W, Hong X, Jiang H and Yu Z: Identification and functional analysis of differentially expressed genes associated with cerebral ischemia/reperfusion injury through bioinformatics methods. Mol Med Rep 18: 1513-1523, 2018.

30. Wang C, Liu M, Pan Y, Bai B and Chen J: Global gene expression profile of cerebral ischemia-reperfusion injury in rat MCAO model. Oncotarget 8: 74607-74622, 2017.

31. Kamme $F$ and Wieloch $T$ : Induction of junD mRNA after transient forebrain ischemia in the rat. Effect of hypothermia. Brain Res Mol Brain Res 43: 51-56, 1996.
32. Sola A, Rogido M,Lee BH,Genetta T and Wen TC: Erythropoietin after focal cerebral ischemia activates the Janus kinase-signal transducer and activator of transcription signaling pathway and improves brain injury in postnatal day 7 rats. Pediatr Res 57: 481-487, 2005.

33. Marden JJ, Zhang Y, Oakley FD, Zhou W, Luo M, Jia HP, McCray PB Jr, Yaniv M, Weitzman JB and Engelhardt JF: JunD protects the liver from ischemia/reperfusion injury by dampening AP-1 transcriptional activation. J Biol Chem 283 6687-6695, 2008.

34. Harari OA and Liao JK: NF-kappaB and innate immunity in ischemic stroke. Ann N Y Acad Sci 1207: 32-40, 2010.

35. Koschmieder S, Rosenbauer F, Steidl U, Owens BM and Tenen DG: Role of transcription factors C/EBPalpha and PU.1 in normal hematopoiesis and leukemia. Int J Hematol 81: 368-377, 2005.

36. Zhang H, Gao W, Qian T, Tang J and Li J: Transcription factor changes following long term cerebral ischemia/reperfusion injury. Neural Regen Res 8: 916-921, 2013.

37. Walton M, Saura J, Young D, MacGibbon G, Hansen W, Lawlor P, Sirimanne E, Gluckman P and Dragunow M: CCAAT-enhancer binding protein alpha is expressed in activated microglial cells after brain injury. Brain Res Mol Brain Res 61: 11-22, 1998.

38. Jin F, Li Y, Ren B and Natarajan R: PU.1 and C/EBP(alpha) synergistically program distinct response to NF-kappaB activation through establishing monocyte specific enhancers. Proc Natl Acad Sci USA 108: 5290-5295, 2011.

39. Formisano L, Guida N, Valsecchi V, Cantile M, Cuomo O, Vinciguerra A, Laudati G, Pignataro G, Sirabella R, Di Renzo G and Annunziato L: Sp3/REST/HDAC1/HDAC2 Complex represses and sp1/HIF-1/p300 complex activates ncx1 gene transcription, in brain ischemia and in ischemic brain preconditioning, by epigenetic mechanism. J Neurosci 35: 7332-7348, 2015.

40. Gelderblom M, Weymar A, Bernreuther C, Velden J, Arunachalam P, Steinbach K, Orthey E, Arumugam TV, Leypoldt F, Simova O, et al: Neutralization of the IL-17 axis diminishes neutrophil invasion and protects from ischemic stroke. Blood 120: 3793-3802, 2012.

41. Tanimura A, Dan S and Yoshida M: Cloning of novel isoforms of the human Gli2 oncogene and their activities to enhance tax-dependent transcription of the human T-cell leukemia virus type 1 genome. J Virol 72: 3958-3964, 1998.

42. Regl G, Kasper M, Schnidar H, Eichberger T, Neill GW, Philpott MP, Esterbauer H, Hauser-Kronberger C, Frischauf AM and Aberger F: Activation of the BCL2 promoter in response to Hedgehog/GLI signal transduction is predominantly mediated by GLI2. Cancer Res 64: 7724-7731, 2004.

43. Zhang L, Chopp M, Meier DH, Winter S, Wang L, Szalad A, Lu M, Wei M, Cui Y and Zhang ZG: Sonic hedgehog signaling pathway mediates cerebrolysin-improved neurological function after stroke. Stroke 44: 1965-1972, 2013.

44. Shi D, Xie F, Zhang Y, Tian Y, Chen W, Fu L, Wang J, Guo W, Kang T, Huang W and Deng W: TFAP2A regulates nasopharyngeal carcinoma growth and survival by targeting HIF-1alpha signaling pathway. Cancer Prev Res (Phila) 7: 266-277, 2014.

45. Lin HH, Chen YH, Chiang MT, Huang PL and Chau LY: Activator protein-2alpha mediates carbon monoxide-induced stromal cell-derived factor-1alpha expression and vascularization in ischemic heart. Arterioscler Thromb Vasc Biol 33: 785-794, 2013.

This work is licensed under a Creative Commons

Attribution-NonCommercial-NoDerivatives 4.0 International (CC BY-NC-ND 4.0) License. 